\title{
Persistence Parameter: a Reliable Measurement for Behavioral Responses of Medaka (Oryzias latipes) to Environmental Stress
}

\author{
Li Yin ${ }^{1} \cdot$ Haitang Yang ${ }^{1} \cdot$ Guiyun $\mathrm{Si}^{1} \cdot$ Qing $\operatorname{Ren}^{1} \cdot \operatorname{Rongshu} \mathrm{Fu}^{1} \cdot \operatorname{Biao} \mathrm{Zhang}^{1}$. \\ Xu Zhang ${ }^{1}$ - Xiaoguang Wang ${ }^{1}$ - Pingping Qi ${ }^{1}$ - Chunlei Xia ${ }^{2}$ - Zongming Ren ${ }^{1}$. \\ Tae-Soo Chon ${ }^{3} \cdot$ Hungsoo $\mathrm{Kim}^{3}$
}

Received: 27 May 2014 / Accepted: 20 March 2015 /Published online: 19 April 2015

(C) Springer International Publishing Switzerland 2015

\begin{abstract}
Online monitoring systems provided a significant evidence for feasibility of the stepwise behavioral response model in detecting the effects of organophosphorus pesticides on movements of medaka (Oryzias latipes), being able to determine the state of indicator organisms, "no effect," "stimulation," "acclimation," "adjustment (readjustment)," and "toxic effect." Though the stepwise behavioral response model postulated that an organism displays a time-dependent sequence of compensatory stepwise behavioral response during exposure to pollutants above their respective thresholds of resistance, it was still a conceptual model based on tendency only in analysis. In this study, the phenomenon of bacterial persistence was used to interpret the relationship between the stepwise behavioral response model and the environmental stress caused by both exposure time and different treatments. Quantitative measurements of the stepwise behavioral response model led to a simple mathematical description of
\end{abstract}

Research Highlights - Measurements of behavior movement led to a simple mathematical description of threshold switch

- "Persister $(p)$ " illustrated behavior "acclimation" and "adjustment (or readjustment)" clearly

- "Persisters $(p)$ " is important for medaka to overcome "threshold" from the environmental stress

Haitang Yang contributed equally to this work.

Zongming Ren

zmren88@gmail.com

1 College of Life Science, Shandong Normal University, Wenhuadong Rd 88, Lixia District Ji'nan 250014, People's Republic of China

2 Yantai Institute of Coastal Zone Research, Chinese Academy of Sciences, Yantai 264003, People's Republic of China

3 Department of Biological Sciences, Pusan National University, Pusan 609735, Republic of Korea the threshold switch, which evaluated the effects of environmental stress on behavioral responses to decide the tendency. The adjustment ability correlated to "persisters $(p)$ " is very important for test individuals to overcome the "threshold" from the outside environmental stress. The computational modeling results suggested that "persister $(p)$," as described in the general equations of bacterial persistence model in changing environments, illustrated behavior acclimation and adjustment (or readjustment) clearly. Consequently, the persistence parameter, $p$, was critical in addressing for medaka to be adapted to fluctuating environments under different environmental stress.

Keywords Stepwise behavioral responses - Oryzias latipes · Persisters $(p) \cdot$ Environmental stress

\section{Introduction}

Motility is a characteristic feature of many organisms that cannot be neglected as an important physiological factor in survival. Behavioral responses in organisms associated with stress and toxicant exposure provide novel information including short-term and median lethal exposure. Meanwhile, behavioral response has been suitable in addressing toxicological impact assessment in all of the test endpoints [1, 8, 14, 18, 32, 35]. By far, behavioral response of various taxa, including crustaceans [41, 25, 7], snails [23, 13], insects [11, 24], and fish [31, 16, $30]$, has been reported to be sensitive to sublethal exposures to various chemical pollutants. Based on the concentration-response-time analysis [43, 19], the assessment of toxicity of chemicals by behavioral eco-toxicology has successfully been applied in Daphnia magna [3]. 
Therefore, it is acceptable to make the prediction of the environmental toxicity based on behavior model analysis.

Due to data complexity, unbalance, and quantity in a huge amount, the behavior movement data are difficult to analyze [10]. Meanwhile, in contrast to genetic variation, which is passed on over many generations, the behavioral responses generated by movement adjustment is difficult to study due to the inherently limited life time of states that are not encoded in the DNA sequence, but makes it possible for the "memory" of past environments to influence future organisms [12]. Sometimes, the commonly used exploratory and statistical modeling techniques may fail to find meaningful ecological patterns from data $[9,40,39]$. However, once a determined behavior movement can be quantified by proper analysis methods, it has the potential to be used as a type of biomarker in the assessment of stress [4].

According to our previous research, the behavioral responses of medaka (Oryzias latipes) exposed in different carbamate pesticides, which included "no effect," "stimulation," "acclimation," "adjustment (readjustment)," and "toxic effect" (Zhang et al., 2004), provided a significant evidence for feasibility of the stepwise behavioral response model. Though the behavior model postulated that an organism displays a time-dependent sequence of compensatory stepwise behavioral response during exposure to pollutants above their respective thresholds of resistance, it was still a conceptual model based on tendency only in analysis. Alternatively, various computational methods, including multi-layer perceptron [28], self-organizing map (SOM) [35], wavelet analysis [36], and fractal dimension [33], have been proposed for objective and automatic analyses of the movement data. Though these methods all played significant roles in the analysis of movement behavior as an indicator in ecological risk assessment, they did not focus on the stepwise behavioral response, covering acclimation, and adjustment in different chemical concentrations. Meanwhile, it is very difficult to deduce the possible behavioral responses only depending on the monitoring the environmental stress. Therefore, more reliable methods should be considered to make mathematical descriptions of the behavioral responses of different individuals to forecast the possible movement tendency without online monitoring of them.

The mathematical analysis of persistence of bacteria in fluctuating environment, described in 2004 [2], allows making a quantitative prediction on evolutionary processes that would promote the emergence of persistence, as well as evaluating its ecological implications. Therefore, the bacterial persistence model, which is a mathematical model to describe the cause of the different sensitive of individuals in one population to the antibiotic, may provide a new quantitative measurement method in modeling the stepwise behavioral response, and the "persister $(p)$ " in persistence model, which was a smart strategy to increase chances of survival of bacterial populations in fluctuating environments [27], may be a good measurement factor of the stepwise behavioral responses of medaka in different treatments.

Therefore, in this study, in order to make a preliminary mathematical description of the stepwise behavioral response model, the behavioral responses of medaka (O. latipes) to three kinds of pesticides (trichlorfon, parathion, and malathion) will be investigated in a monitoring system. Based on the conceptual description of the behavioral responses, the persister $(p)$ in persistence model will be used to measure the behavior acclimation and behavior adjustment (or readjustment).

\section{Methods and Model}

\subsection{Experimental Design}

\subsubsection{Test Species and Chemicals}

The individuals of medaka fish were provided by the Research Center for Eco-Environmental Sciences, Chinese Academy of Sciences (Beijing, China). The brood stock was raised in flowthrough system with dechlorinated tap water (using active carbon) at a temperature of $20 \pm 2{ }^{\circ} \mathrm{C}$. The photoperiod was maintained with 16-h light (04:00-20:00, with 4000 lux light intensity) and 8-h dark (20:00-04:00, no light given). The brood stock was fed with newly hatched brine shrimp in the morning (7:00) and flake food (Trea ${ }^{\circledR}$, Germany) in the afternoon (18:00). Fifteen days after hatching, medaka was fed two times every day with flake food. Medaka individuals about $2.5 \sim 3.0 \mathrm{~cm}$ long ( 2 months after hatching) were used for test organisms.

Trichlorfon $(T)$, parathion $(P)$, and malathion $(M)$ were purchased from J\&K Chemical Ltd. All compounds were of technical grade ( $>95 \%$ purity). Stock solutions (stored at $4{ }^{\circ} \mathrm{C}$ until use) with proper concentrations were prepared with dimethyl sulfoxide as a solvent and were diluted with appropriate aliquots. All solvents were of analytical grade. The concentration of dimethyl sulfoxide used for test was less than $0.5 \%$ in all experiments, which would neither lead to acute toxicity to medaka nor affect the mobility [42].

\subsubsection{Monitoring of Medaka Behavior Movement}

Behavioral response monitoring was carried out under flowthrough conditions by an online monitoring system (OMS) built in Chinese Academy of Sciences. Test organisms were placed in a flow-through test chamber $(7 \mathrm{~cm}$ long, $5 \mathrm{~cm}$ in diameter), which was closed off with nylon nets $(250 \mu \mathrm{m})$ on both sides [38]. One pair of electrodes located at the inside walls of the test chambers and sent a high frequency signal of altering current, which was received by a second pair of noncurrent-carrying electrodes located at the inside walls 
[47]. Overall response activity of test organisms inside the chamber is reflected on the signal and is transformed by the $\mathrm{A} / \mathrm{D}$ converter. Detailed mechanism in OMS can be referred to Ren and Wang [38]. We define the converted data in OMS as behavior strength in the chamber, and the data present the behavioral state responding to environmental stimuli [38]. Behavior strength was automatically sampled by OMS in each second, and average values in every 6 min were produced as output. Values of behavior strength were normalized, ranging from 0 (loss of motility) to 1 (full behavior express).

Three healthy medaka individuals were selected at random for each test chamber, and three test chambers were used for each treatment. The flow rate of each test chamber was controlled about $2 \mathrm{~L} / \mathrm{h}$, which was no effect on the motility of test organisms [17]. Observation started in 16:00 and finished in 16:00 of the third day for all chambers. During observation period, no food was provided to test medaka. Light and temperature conditions were the same as given in rearing stock populations.

Chemical toxic unit (TU) to test organisms was used for comparison of toxic effects. The 48-h median lethal concentration $\left(\mathrm{LC}_{50-48}\right)$ was taken as one unit (1 TU) [26]. The calculation of TU value is shown as follows:

$\mathrm{TU}=\frac{C_{i}}{\mathrm{LC}_{50-48}^{i}}$

where $C_{i}$ was the concentration of chemical $i$ and $\mathrm{LC}_{50-48}{ }^{i}$ was the 48-h median lethal concentration. Sublethal concentration was regarded as $10 \% \mathrm{LC}_{50-48}$ [26], which was about $0.1 \mathrm{TU}$. Four concentrations of $0.1,1,5$, and $10 \mathrm{TU}$ were tested with each chemical. Based on the previous reports $[34,46]$, the $\mathrm{LC}_{50-48}$, which was regarded as $1 \mathrm{TU}$ in this study, was 10 , 2.9 , and $0.75 \mathrm{mg} / \mathrm{L}$ for $T, P$, and $M$, respectively.

\subsection{Persistence Model}

According to previous research, a fraction of a genetically homogeneous microbial population may survive exposure to stress such as antibiotic treatment [22]. Unlike resistant mutants, cells regrown from such persistent bacteria remain sensitive to the antibiotic [2]. Several hypotheses have been put forward in order to explain the heterogeneous response (persistence) to antibiotics in apparently uniform populations [29]. Already in 1944, Bigger had suggested that "persisters $(p)$ " are in a dormant state $[5,6]$. It has also been proposed, for instance, that persistent bacteria are those that find themselves in some "protected" part of the cell cycle at the time of exposure to antibiotics. Then, it is defined that persistence is a characteristic of a heterogeneous bacterial population that is taken as a generic example through which the dynamics of population variability could be investigated [15].
As descript in 2004 by Balaban [2], due to the characteristics of different bacteria, two types (type I and type II) of mathematical description of persistence were made. Type I persisters $(p)$ are generated by trigger events during stationary phase (Eq. 2), whereas type II persisters ( $p$ ) are continuously generated during growth (Eq. 3).

$\left\{\begin{array}{l}\frac{d p_{\mathrm{I}}}{d t}=-b p_{\mathrm{I}}+\mu_{p} p_{\mathrm{I}} \\ \frac{d n}{d t}=b p_{\mathrm{I}}+\mu_{n} n\end{array}\right.$

$\left\{\begin{array}{l}\frac{d n}{d t}=-a n+b p_{\mathrm{II}}+\mu_{n} n \\ \frac{d p_{\mathrm{II}}}{d t}=a n-b p_{\mathrm{II}}+\mu_{p} p_{\mathrm{II}}\end{array}\right.$

The general equations described the dynamics of two subpopulations, which denoted as normal $(n)$ and persister $(p)$ cells. The growth-death rates of the persisters $(p)$ and the normally growing cells were characterized in the persistence model by the constants $p$ and $n$, respectively. The individuals switched from the $n$ state to the $p$ state with a constant rate $a$ or from the $p$ state to the $n$ state with a constant rate $b$.

The quantitative measurements of the key parameters that characterize persistence make possible the discrimination between seemingly similar persistence phenotypes. Persistence could be reduced with factors that affect the lag period, whereas spontaneous persistence could be targeted by factors specifically enhancing the switching rate from persister $(p)$ to normal cells $(n)$. Therefore, in the process of ecology and evolution, persistence could be regarded as a survival strategy for individuals in fluctuating environments, and persisters $(p)$ play an important role in the strategy.

\section{Results and Discussion}

\subsection{The Effects of OPs on Medaka Behavior Movement}

The behavior data was analyzed by a 3-D Surface Plot of MATLAB 2009 (C 1984-2009 The MathWorks, Inc.). Surf $(X, Y, Z)$ creates a shaded surface using $Z$ for the data as well as surface height. $X$ and $Y$ are vectors defining the $x$ and $y$ components of a surface. Length $(X)=n$ and length $(Y)=m$, where $[m, n]=$ size $(Z)$. In this case, the vertices of the surface faces are $(X(j), Y(i), Z(i, j))$ triples.

After the 3-D surface analysis, the changes in behavior strength (BS) after exposed to $T, P$, and $M$ are shown as the exposure time progress in Fig. 1. The BS values in the control were stable about 0.8 . This value was also observed at the initial period of every exposure (the first $6 \mathrm{~min}$ ). When test organisms were exposed to minimal concentration of $0.1 \mathrm{TU}$ (Fig. 1), overall response patterns were similar in different chemicals. With higher concentrations, the BS values 
Fig. 1 The effects of different treatments on medaka behavioral responses in different time. Exposure time was showed as log value, and BS values were used to statistic the tendency of medaka BR in different OPs. $T, P$, and $M$ meant the effects of trichlorfon, parathion, and malathion separately on medaka BR

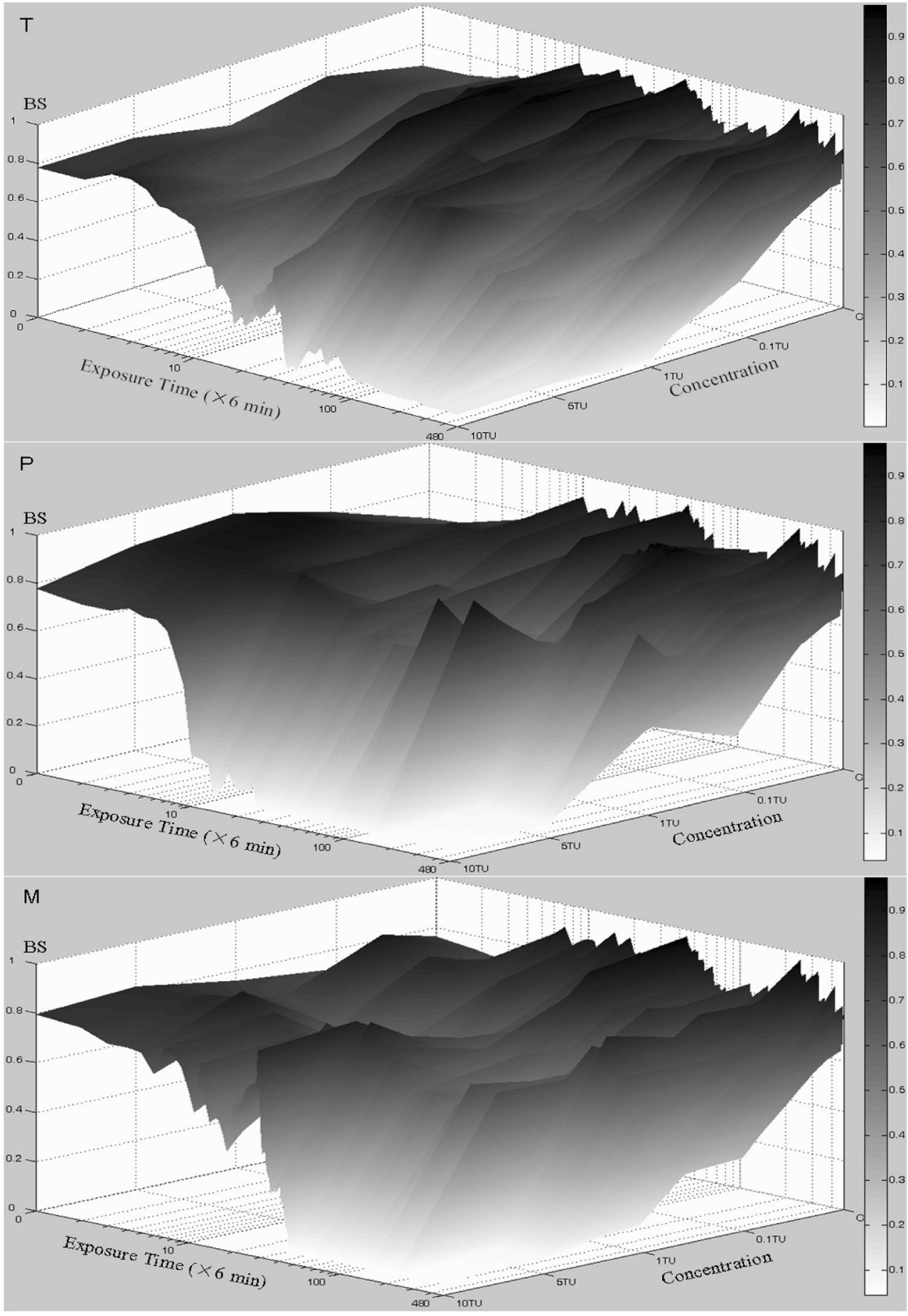

decreased more strongly and impact occurred in the earlier period after exposure to chemicals. In the same treatment of concentration, BS decreased along with exposure time although there were some exceptions (i.e., temporary recovery) during the course of stepwise response to chemicals. When toxic treatment was minimal with $0.1 \mathrm{TU}, \mathrm{BS}$ varied as the time progress, including recovery and declination for a few times during the observation period. This illustrated that behavioral response was stepwise modulation with adjustments and readjustments.

Occurrence of adjustments and readjustments decreased as TU increased. With maximal $10 \mathrm{TU}, \mathrm{BS}$ decreased rapidly around exposure time $6 \mathrm{~min}$ for all chemicals. These results suggested that BS expressed different environmental stress. In lower concentration treatments, e.g., 0.1 and 1 TU, medaka BS SBR was more significant, which mainly contained stimulation, acclimation, and adjustment (readjustment), without 
toxic effect. In 5 TU OP treatments during the exposure period, medaka BR met a great many changes, which mainly included stimulation, acclimation, adjustment (readjustment), and toxic effect [37]. In the highest concentration exposure, it was hard for medaka to have the capability of adjustment.

Based on analysis of the effects of the three kinds of organophosphorus pesticides on medaka stepwise behavioral responses in time series (Fig. 2a), the behavior strength showed evident time-dependent decrease, followed by a second and a third increase in lower concentration treatments as illustrated by the thick dotted curves I and II. These results suggested that the stepwise behavioral responses were more significant than in10 TU treatments (III), which showed no more adjustment than in lower concentration treatments. As shown in Fig. 2, the medaka stepwise behavioral responses were illustrated clearly by the tendency curves (I, II, and III). Higher toxic effects on behavior movement induced BS decrease as curve III with hardly any adjustment, and in lower environmental stress, behavioral response tendency could be reflected by curves I and II, which included behavior stimulation, acclimation, adjustment, and toxic effects as described by Selye [44]. Though number of the treatment groups was quite less than time series data, the analysis of BS changes in the treatments series (Fig. 2b) could show the similar tendency as Fig. 2a, which suggested that the environmental stress will increase due to the increase of treatment concentration of chemicals in the same exposure time.

In weaker environmental stress, e.g., 0.1 and $1 \mathrm{TU}$ in time series, medaka stepwise behavioral responses were more significant, which mainly contained stimulation, acclimation, and adjustment (readjustment), without toxic effect. In 5 TU treatments during the exposure period, medaka behavioral responses met a great many changes, which mainly included stimulation, acclimation, adjustment (readjustment), and toxic effect. In the highest concentration exposure (10 TU), it was hard for medaka to have the capability of adjustment. Medaka behavioral responses decreased with hardly any movement modulation, and the reason for this might be that in the higher concentration exposure, the intrinsic responses were not strong enough to adjust inner environment by behavioral responses to adapt the outside stress [45]. The stepwise behavioral responses of medaka to the three kinds of organophosphorus pesticides could be described as the conceptual model as shown in Fig. 3 [48].

\subsection{Analysis of the Behavioral Responses Based on Persister ( $p$ )}

The stepwise behavioral responses of medaka to the three kinds of organophosphorus pesticides showed evident regularity, which was illustrated in the conceptual stepwise behavioral response model [48]. Behavior stimulation was usually followed by acclimation which was a continuous weakening
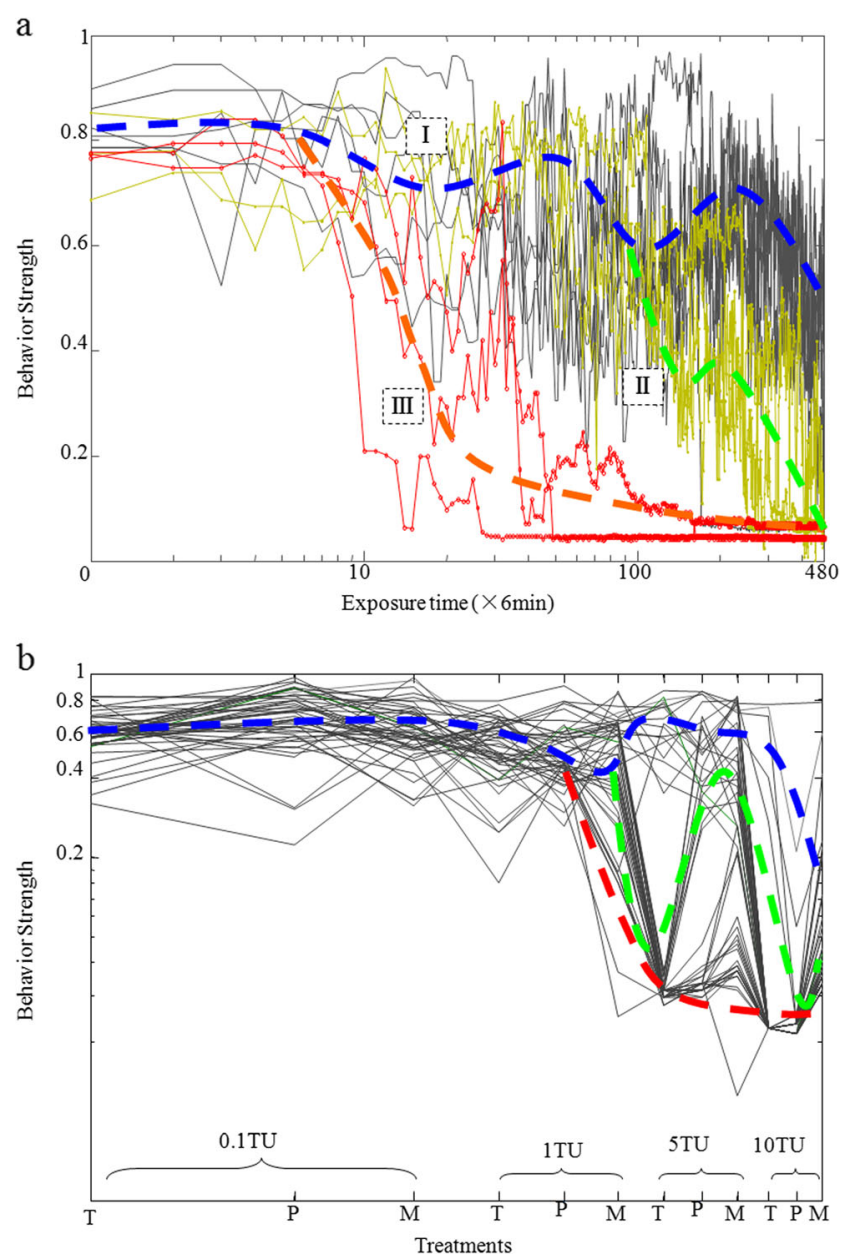

Fig. 2 The tendency analysis of the effects of organophosphorus pesticides on medaka stepwise behavioral responses under environmental stress. The tendency analysis of the stepwise behavioral responses focused on the total changes of the behavior strength. a Solid curves showed the BS changes in time series. The red solid curves showed the BS changes in $10 \mathrm{TU}$ treatments, the green solid curves showed the BS changes in 5 TU treatments, and the gray solid curves showed the BS changes in other concentration treatments ( 1 and $0.1 \mathrm{TU}$ ). The thick-dotted curves (I, II, and III) in figure meant the tendency of medaka stepwise behavioral responses in different kinds of treatments. b Gray solid curves showed BS changes in the treatments series every 10 min. $T, P$, and $M$ mean trichlorfon, parathion, and malathion, respectively. The thick-dotted curves meant the tendency of medaka stepwise behavioral responses in different time

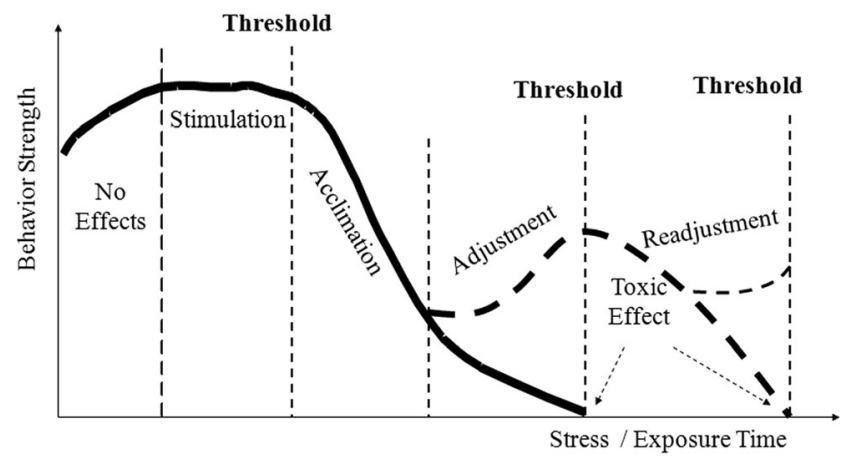

Fig. 3 Stepwise behavioral responses of medaka to environmental stress 
of BS. The main reason for the fall of movement behavior in acclimation was that the behavior adjustment reached extreme of "alarm reaction" due to "threshold" in Fig. 3. However, the threshold caused by the outside environmental stress is not enough to clear the model of behavioral responses. According to the "persistence model," sometimes, the situation of the individuals living in different environments may play a significant role in maintaining their own behavior movement ability.

Bacteria can protect themselves from various stresses at the cost of suspending their growth, and the slow-growing persister $(p)$ cells can save the population from extinction during times of stress $[2,27]$. Therefore, the actual investment in such an insurance policy, namely, the number of persister $(p)$, is known to be genetically determined and could therefore be subject to evolutionary adaptation.

As reported by Harrison $[21,20]$, the bacteria can be maintained in the environment owing to co- or cross-resistance to toxic chemicals (metals) or the regulation of resistance pathways, which highlights the importance of persisters $(p)$ as shown in Fig. 4. The number of individuals will decrease slowly during the exposure process, which includes resistence, inhibition, decrease, and then persistence (tolerance). It is hypothesized that once there were no strong treatments, the change trends of the individual number might be shown as the dotted curve in Fig. 4, and the process will be quite similar to the stepwise behavioral response model as described in Fig. 3. Therefore, in the aspect of the individual situation, the adjustment ability correlated to persisters $(p)$ is very important for them to overcome the threshold from the outside environmental stress.

According to the persistence model, the process of the stepwise behavioral responses of medaka to organophosphorus pesticide is also a living strategy to protect them from the negative impact of the environmental stress. Persisters $(p)$ of behavior movement may help individuals complete the acclimation and "behavior adjustment," and it is a reliable measurement for behavioral responses of medaka to environmental stress.

\subsection{Preliminary Mathematical Description of the Behavioral Responses Based on Persister (p)}

The immobility of medaka will cause the movement toxic effects in the stepwise behavioral response model; however, the number of bacteria individuals will not decrease to zero due to persisters $(p)$. Though there is evident difference between the two models, the measurement of the stepwise behavioral responses model based on persisters $(p)$ is acceptable, especially in the process of behavior acclimation and "adjustment." Therefore, in this study, the preliminary mathematical description of the medaka stepwise behavioral responses based on persister $(p)$ is developed.

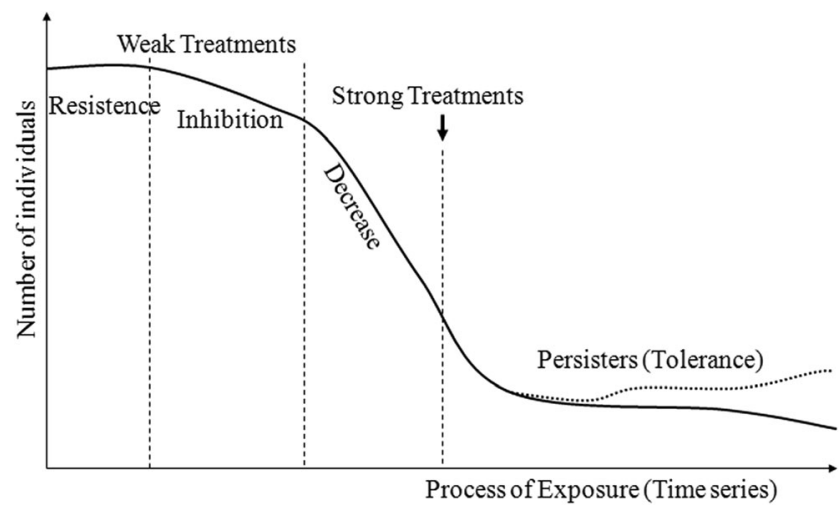

Fig. 4 The importance of "persisters ( $p$ )" cells in the process of exposure in different chemicals. The dotted curve during the tolerance period shows the possible tendency of the individuals once there are no strong treatments in the exposure process

In the mathematical description of the medaka stepwise behavioral responses, $y(t)$ is the macroscopic (phenomenological) presenting of BS without considering the differences among different individuals and the effects of the other environmental factors. During the periods of no effects, stimulation, and acclimation, the normal behavior $\left(B_{n}\right)$ decides the trends of BS changes. The environmental stress $\left(S_{e}\right)$ due to both the same exposure time in different treatments and the same treatment in different exposure time will inhibit the normal behavior. In the process of adjustment and readjustment, the persister $(p)$ behavior $\left(B_{p}\right)$ is the dominant. With the analysis of both Figs. 3 and 4, the appearance of persister $(p)$ is always following the threshold from the environmental stress, which means that the persister $(p)$ behavior could be induced by the threshold of environmental stress $\left(S_{T}\right)$. Once behavior toxic effects start $\left(T_{b}\right)$, there will be no $S_{T}$ again, and the persister $(p)$ behavior $B_{p}$ will not be excited but decrease slowly as $\left(B_{0}\right)$ until the lost of behavior movement. The schematic changes curves of different stepwise behavioral responses of medaka are shown in Fig. 5.

Normally, $y(t)$ is equal to the normal behavior $B_{n}$ in pollution-free environment as shown in Eq. 4. The $B_{n}$ curve recorded by online monitoring system shows some fluctuation in Fig. 5a, which means that $y(t)$ value is constantly changing in a certain range.

$y(t) s=B_{n}$

Once subjected to environmental stress, the stepwise behavioral responses $y(t)$ will be decided by both $B_{n}$ and $B_{p}$ (Eq. 5), which are illustrated by the dotted black curves in Fig. 5b.

$y(t)=B_{n}+B_{p}$

There is no direct correlation between $B_{n}$ and $B_{p}$, but they will be affected by environmental stress. Under the conditions 
Fig. 5 Schematic change curves of different stepwise behavioral responses of medaka

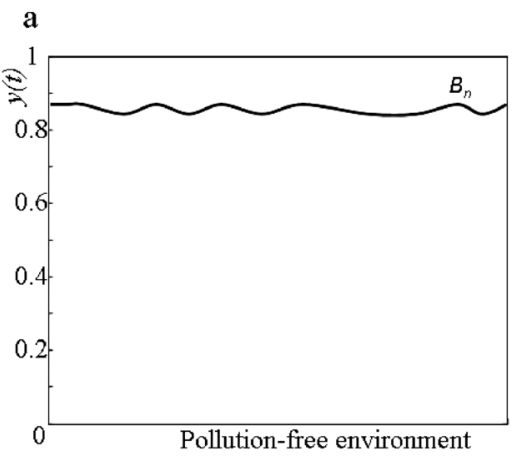

of environmental pollution, there is a negative correlation between $B_{n}$ and $S_{e}$ (Eq. 6).

$f\left(B_{n}\right)=f^{-1}\left(S_{e}\right)$

For $B_{p}$, the persister $(p)$ behavior will be induced by $S_{e}$, especially when $S_{e}$ reaches the threshold of environmental stress $\left(S_{T}\right)$ (Eq. 7). It is hypothesis that there are $i$ thresholds during the exposure of medaka in different treatments, and then the medaka stepwise behavioral responses $y(t)$ will be presented as Eq. 8.

$f\left(B_{p}\right)=f\left(S_{T}\right) * f\left(S_{e}\right)$

$y(t)=\sum_{i=1}^{i} B_{p i}$

Meanwhile, once behavior toxic effects start $\left(T_{b}\right)$, there will be no $S_{T}$ again, and $B_{p}$ will be $B_{0}$ (Eq. 9). In this case ( $\left.B_{0}\right)$, even test individuals (medaka) are moved to pollution-free environment, it cannot stop $B_{p}$ to decrease to zero (Fig. $5 \mathrm{~b}$ ).

$f\left(B_{p}\right)=f\left(B_{0}\right)$

The model of the stepwise behavioral responses based on persister $(p)$ as showed above is simple, but it is the first time to give a preliminary mathematical description based on BS, which is monitored by an online monitoring system. This mathematical description suggested that persister $(p)$ based on the bacterial "persistence model" could help test individuals overcome the threshold from the outside environmental stress, and these equations about the stepwise behavioral responses could illustrate different movement process of test medaka under environmental stress and supply a good method to clear the behavior trend analysis.

\section{Conclusions}

The phenomenon of bacterial persistence was observed as early as in 1942, when Staphyloccocal infections were seen to recur, even following extensive treatments with high doses of penicillin [22], and the first conceptual description of threshold environmental stress was discussed in 1973 [44]. In this study, the mathematical description of the stepwise behavioral response model to environmental stress based on bacterial persistence is developed for the first time, which might be very helpful for us to illustrate or postulate the possible behavior tendency according to the environmental stress.

The persister $(p)$ according to persistence model is a reliable measurement for medak under environmental stress. Especially when the threshold of environmental stress inhibits the behavior movements, persister $(p)$ behavior will help individuals complete the acclimation and behavior adjustment to overcome the outside environmental threat.

However, the threshold switch between normal behavior movement and the behavior adjustment was found to depend strongly on the environmental stress but weakly on the exposure time of any given treatments due to the less number of the treatment groups. Therefore, further research would focus on the treatment series exposure effects on the behavior movement of medaka, and behavioral responses of other aquatic organisms, e.g., crustaceans, snails, insects, and other fish, to the other different chemicals should be investigated to develop the mathematical stepwise behavioral response model.

Meanwhile, the mathematical description of the stepwise behavioral response model is just started, and the simple equations showed in this study are not enough to satisfy the necessary of the behavior tendency analysis based on the environmental stress. Some other mathematical methods, e.g., some neural network model to realize the complexity analysis of behavior data and Kalman Filter methods to filtering behavior trends should be applied to refine the stepwise behavioral response model.

Acknowledgments This study was financially supported by the National Natural Science Foundation of China (21107135). 


\section{References}

1. Azizullah, A., Richter, P., \& Häder, D. P. (2011). Comparative toxicity of the pesticides carbofuran and malathion to the freshwater flagellate Euglena gracilis. Ecotoxicology, 20(6), 1442-1454.

2. Balaban, N. Q., Merrin, J., Chait, R., Kowalik, L., \& Leibler, S. (2004). Bacterial persistence as a phenotypic switch. Science, 305, 1622-1625.

3. Barata, C., Baird, D. J., \& Markich, S. J. (1999). Comparing metal toxicity among Daphnia magna clones: an approach using concentration-response-time surfaces. Archives of Environmental Contamination and Toxicology, 37, 326-331.

4. Beitinger, T. (1990). Behavioral reactions for the assessment of stress in fishes. Journal of Great Lakes Research, 16, 495-528.

5. Bigger, J. W. (1944). The bactericidal action of penicillin on Staphylococcus pyogenes. Irish Journal of Medical Science, 227, 553-568.

6. Bigger, J.W. (1944b). Treatment of staphylococcal infections with penicillin by intermittent sterilisation. Lancet, 2, 497-500.

7. Billoir, E., Alexandre, R. R. P., \& Charles, S. (2007). Integrating the lethal and sublethal effects of toxic compounds into the population dynamics of Daphnia magna: a combination of the DEBtox and matrix population models. Ecological Modelling, 203, 204-214.

8. Bracy, O. L., Doyle, R. S., Kennedy, M., McNally, S.M., Weed, J. D., \& Thorne, B. M. (1979). Effects of methomyl and ethanol on behavior. In The Sprague-Dawley Rat. Pharmacology Biochemistry and Behavior, 10(1), 21-25.

9. Breiman, L., Friedman, J. H., Olshen, R. A., \& Stone, C. G. (1984). Classification and regression trees. Belmont: Wadsworth International Group.

10. Cho, E., \& Chon, T. (2004). Application of wavelet analysis to ecological data. Ecological Informatics, 2006, 1(3), 229-233.

11. Chon, T., Park, Y. S., \& Ross, M. H. (1998). Activity of German cockroach, Blattella germanica (L.) (Orthoptera: Blattellidae), at different microhabitats in semi-natural conditions when treated with sublethal doses of pesticides. Asia-Pacific Entomology, 1, 77-83.

12. Clayton, N. S., Bussey, T. J., \& Dickinson, A. (2003). Can animals recall the past and plan for the future? Nature Reviews Neuroscience, 4, 685-691.

13. Coffin, M. R. S., Drolet, D., Hamilton, D. J., \& Barbeau, M. A. (2008). Effect of immersion at low tide on distribution and movement of the mud snail, Ilyanassa obsoleta (Say), in the upper Bay of Fundy, eastern Canada. Journal of Experimental Marine Biology and Ecology, 364, 110-115.

14. Farr, A. J., Chabot, C. C. T., \& Douglas, H. (1995). Behavioral avoidance of fluoranthene by fathead minnows (Pimephales promelas). Neurotoxicology and Teratology, 17, 265-271.

15. Gefen, O., \& Balaban, N. Q. (2009). The importance of being persistent: heterogeneityof bacterial populations underantibiotic stress. FEMS Microbiology Reviews, 33, 704-717.

16. Gray, M. A., Teather, K. L., \& Metcalfe, C. D. (1999). Reproductive success and behavior of Japanese medaka (Oryzias latipes) exposed to 4-tera-octylphenol. Environmental Toxicology and Chemistry, $18,2587-2594$.

17. Guilhermino, L., Diamantino, T., Silva, M. C., \& Soares, A. M. V. M. (2000). Acute toxicity test with Daphnia magna: an alternative to mammals in the prescreening of chemical toxicity. Ecotoxicology and Environmental Safety, 46, 357-362.

18. Hanazato, T., \& Fueki, K. (2001). Fish-induced life-history shifts in the cladocerans Daphnia and Simocephalus: are they positive or negative responses. Journal of Plankton Research, 23(9), 945-951.

19. Hanson, M. L., \& Solomon, K. R. (2002). New technique for estimating thresholds of toxicity in ecological risk assessment. Environmental Science and Technology, 36, 3257-3264.
20. Harrison, J. J., Ceri, H., \& Turner, R. J. (2007). Multimetal resistance and tolerance in microbial biofilms. Nature Reviews Microbiology, 5, 928-938.

21. Harrison, J. J., Turner, R. J., \& Ceri, H. (2005). Persister cells, the biofilm matrix and tolerance to metal cations in biofilm and planktonic Pseudomonas aeruginosa. Environmental Microbiology, 7, 981-994.

22. Hobby, G. L., Meyer, K., \& Chaffee, E. (1942). Observations on the mechanism of action of penicillin. Proceedings of the Society for Experimental Biology and Medicine, 50, 281-285.

23. Ibrahim, W. L. F., Furu, P., Ibrahim, A. M., \& Christensen, N. O. (1992). Effect of the orgaophosphorous insecticide, chlorpyrifos (Dursban), on growth, fecundity and mortality of Biomphalaria aexandrina and on the production of Schistosoma mansoni cercariae in the snail. Journal of Helminthology, 66, 79-88.

24. Ji, C., Lee, S., Choi, K., Kwak, I., Lee, S., Cha, E., Lee, S., \& Chon, T. (2007). Monitoring of movement behaviors of chironomid larvae after exposure to diazinon using fractal dimension and selforganizing map. International Journal of Ecodynamics, 2(1), 27-38.

25. Kim, C., Kwak, I., Cha, E., \& Chon, T. (2006). Implementation of wavelets and artificial neural networks to detection of toxic response behavior of chironomids (Chironomidae: Diptera) for water quality monitoring. Ecological Modelling, 195, 61-71.

26. Kong, F., Yin, D., \& Yan, G. (2000). Environmental biology. Beijing: Higher Education Press (In Chinese).

27. Kussell, E., Kishony, R., Balaban, N. Q., \& Leibler, S. (2005). Bacterial persistence: a model of survival in changing environments. Genetics, 169, 1807-1814.

28. Kwak, I., Chon, T., Kang, H., Chung, N., Kim, J., \& Koh, S. (2002). Pattern recognition of the movement tracks of medaka (Oryzias latipes) inresponse to sub-lethal treatments of an insecticide by using artificial neural networks. Environmental Pollution, 120, 671-681.

29. Lewis, K. (2000). Programmed death in bacteria. Microbiology and Molecular Biology Reviews, 64, 503-514.

30. Liu, Y., Lee, S., \& Chon, T. (2011). Analysis of behavioral changes of zebrafish (Danio rerio) in response to formaldehyde using selforganizing map and a hidden Markov model. Ecological Modelling, 222(14), 2191-2201.

31. Moore, A., \& Waring, C. P. (1996). Sublethal effects of the pesticide diazinon on olfactory function in mature male Atlantic salmon parr. Journal of Fish Biology, 48, 758-775.

32. Nakayamaa, K., Oshimaa Y., Tsurudab, Y., Kanga, I. J., Kobayashic, M., Imadaa, N., \& Honjo, T. (2004). Fertilization success and sexual behavior in male medaka, Oryzias latipes, exposed to tributyltin. Chemosphere, 55(10), 1331-1337.

33. Nimkerdphol, K., \& Nakagawa, M. (2008). Effect of sodium hypochlorite on zebrafish swimming behavior estimated by fractal dimension analysis. Journal of Bioscience and Bioengineering, 105(5), 486-492.

34. Nishiuchi, Y., \& Hashimoto, Y. (1967). Toxicity of pesticide ingredients to some fresh water organisms. Science of Pest Control, 32, 5-11.

35. Park, Y., Chung, N., Choi, K., Cha, E., Lee, S., \& Chon, T. (2005). Computational characterization of behavioral response of medaka (Oryzias latipes) treated with diazinon. Aquatic Toxicology, 71, 215-228.

36. Polansky, L., Wittemyer, G., Cross, P. C., Tambling, C. J., \& Getz, W. M. (2010). From moonlight to movement and synchronized randomness: Fourier and wavelet analyses of animal location time series data. Ecology, 91(5), 1506-1518.

37. Ren, Z., Li, Z., Zha, J., Ma, M., Wang, Z., \& Gerhardt, A. (2007). The early warning of aquatic organophosphorus pesticide contamination by on-line monitoring behavioral changes of Daphnia magna. Environmental Monitoring and Assessment, 134, 373-383. 
38. Ren, Z., \& Wang, Z. (2010). The differences in the behavior characteristics between Daphnia magna and Japanese madaka in an online biomonitoring system. Journal of Environmental Sciences, 22(5), 703-708.

39. Richard, O. D., Peter, E. H., \& David, G. S. (2001). Pattern classification 2nd edn. USA: Wiley Interscience.

40. Ripley, B. D. (1996). Pattern recognition and neural networks. Cambridge: Cambridge University Press.

41. Roast, S. D., Widdows, J., \& Jones, M. B. (2000). Disruption of swimming in the hyperbenthic mysid Neomysis integer (Peracarida: Mysidaces) by the organophosphate pesticide chlorpyrifos. Aquatic Toxicology, 47, 227-241.

42. Sandbacka, M., Christianson, I., \& Isomaa, B. (2000). The acute toxicity of surfactants on fish cells, Daphnia magna and fish-a comparative study. Toxicology in Vitro, 14, 61-68.

43. Scholze, M., Bodeker, W., Faust, M., Backhaus, T., Altenburger, R., \& Grimme, L. H. (2001). A general best-fit method for concentrationresponse curves and the estimation of low-effect concentrations. Environmental Toxicology and Chemistry, 20(2), 448-457.
44. Selye, H. (1973). The evolution of the stress concept. American Scientist, 61, 692-699.

45. Sun, R. Y., Li, B., Zhuge, Y., \& Shang, Y. C. (1993). General ecology. Beijing: Higher Education Press.

46. Tsuda, T., Kojima, M., Harada, H., Nakajima, A., \& Aoki, S. (1997). Acute toxicity, accumulation and excretion of organophosphorous insecticides and their oxidation products in killifish. Chemosphere, 35(5), 939-949.

47. Wang, Y., Liu, X., Hu, J., Li, L., \& Li, Y. (2007). The simulation and experimental study of conductance sensor's electric field distribution. Petrol Instruments, 1(21), 16-18 (in Chinese).

48. Zhang, G., Chen, L., Chen, J., Ren, Z., Wang, Z., \& Chon, T.-S. (2012). Evidence for the stepwise behavioral response model (SBRM): the effects of carbamate pesticides on medaka (Oryzias latipes) in an online monitoring system. Chemosphere, 87, 734-741. 